\title{
Real-time Characterization of Chemical Structure and Dynamics of Electrical Double Layer at Electrode-electrolyte Interfaces
}

Yanyan Zhang $^{a}$, Jilin Tang $^{a, b}$, Zhigang $\mathrm{Ni}^{c}$, Yao Zhao $^{a}$, Feifei Jia ${ }^{a}$, Qun Luo $^{a, b}$, Lanqun Mao $^{a, b}$, Zihua Zhu ${ }^{d, *}$, Fuyi Wang $^{a, b, *}$

${ }^{\text {a }}$ Beijing National Laboratory for Molecular Sciences, National Centre for Mass Spectrometry in Beijing, CAS Key Laboratory of Analytical Chemistry for Living Biosystems, Institute of Chemistry, Chinese Academy of Sciences, Beijing 100190, China.

${ }^{\mathrm{b}}$ University of Chinese Academy of Sciences, Beijing 100049, China.

${ }^{c}$ College of Materials, Chemistry and Chemical Engineering, Hangzhou Normal University, Hangzhou 311121, China.

d Environmental Molecular Sciences Laboratory, Pacific Northwest National Laboratory, Richland, WA, 99354, USA

AUTHOR INFORMATION

\section{Corresponding Author}

*Fuyi.Wang: fuyi.wang@iccas.ac.cn. *Zihua Zhu: zihua.zhu@pnnl.gov. 
ABSTRACT. The chemisorption of species from supporting electrolytes on electrode surfaces is ubiquitous in electrochemical systems and affects the dynamics and mechanism of various electrochemical reactions. The understanding of chemical structure and property of the resulting electrical double layer is vital but limited. In this work, we operando probed the electrochemical interface between a gold electrode surface and a common supporting electrolyte, phosphate buffer, using our newly developed in situ liquid secondary ion mass spectrometry during dynamic potential scanning. We surprisingly found that on the positively charged gold electrode surface sodium cations coexisted within the inner Helmholtz layer to form ion pairs with the accumulated phosphate anions, resulting in a strong and dense adsorption phase which was further revealed to retard the electro-oxidation reaction of ascorbate. This finding addressed one major gap in the fundamental science of the electrode-electrolyte interface that where and how the cations exactly reside in the double layer to impose effects on electrochemical reactions, providing insights into engineering of better electrode-electrolyte interfaces in a wide range of fields such as electrochemical conversion and storage of energy, electrocatalysis, and electrodeposition. 
Electrochemistry is central to a plethora of fields including energy conversion and storage, biochemistry, sensors and corrosion, and concerned with the dynamic structure and processes at the electrode-electrolyte interface. For the knowledge-based development of these areas, fundamental understanding of the corresponding electrode-electrolyte interfaces, particularly of the interface structure and dynamics on the molecular scale, is greatly demanded. ${ }^{1-5}$ As first realized by Helmholtz, when an electrode is electrically charged it repels ions with the same sign and attracts those with opposite charge from the electrolyte, forming a so-called electrical double layer at the interface between the electrode and electrolyte. ${ }^{6}$ However, for systems involving the chemisorption of species from electrolytes on electrode surfaces, which is ubiquitous and has been of great concern in electrochemistry, this classical double layer theory is challenged and far away from fully describing the structure and dynamics at the interface.

One of such common examples exists in the systems of using phosphate buffer as the supporting electrolytes, which are the most prevalent media for controlling $\mathrm{pH}$ over a wide range for both fundamental researches and practical applications in fields of (bio)electrochemistry, electro-catalysis and sensors ${ }^{7-13}$. The strong adsorption of phosphate anions at metal electrode surfaces can greatly alter the electrode surface chemistry and affect the dynamics and mechanism of a wide range of electrochemical processes. ${ }^{9,11}$ With regard to this, unravelling of the double layer structure and its influence on the interface properties are currently hot topics in the fields of electrochemistry. The depicting of this picture has to tackle with the nanoscale presence of the double layer, the dynamic electrode potential during realistic electrochemical conditions and the complicated electrochemical interface in the multicomponent buffer as well. In situ studies based on vibrational spectroscopy methods, particularly infrared reflection adsorption spectroscopy $(\text { IRAS })^{14-16}$ and surface enhanced Raman spectroscopy (SERS) ${ }^{17,18}$, have provided valuable 
information on the local chemical bonding and orientation of adsorbed anion species at the interface. However, an accurate and comprehensive characterization of the dynamic double layer structure describing where and how both the anions and cations exactly reside at the interface to impose effects on electrochemical reactions under operando conditions is still absent.

Herein, we employed the recently developed in situ liquid secondary ion mass spectrometry (SIMS) technique ${ }^{19-28}$ to address the issues mentioned above. With the merit of high spatial and temporal resolution, the liquid SIMS has convincingly demonstrated the classic double layer theory in relatively simple systems of electrolytes. ${ }^{20,24,28}$ In this work, we chose a commonly used multicomponent electrolyte, the neutral phosphate buffer $\left(\mathrm{NaH}_{2} \mathrm{PO}_{4}-\mathrm{Na}_{2} \mathrm{HPO}_{4}\right)$, and utilized the in situ liquid SIMS approach to unravel the dynamic structure of the much more complex electrode-electrolyte interface when adsorption of phosphate anions were expected at a dynamically electrified gold electrode surface. Surprisingly, sodium cations were found to coexist in the inner Helmholtz layer at an anodic polarized gold surface and play a vital role in forming the strong and dense adsorption phase through cation-anion interactions with the accumulated phosphate anions. With further studies in such a system containing ascorbate we unraveled that it is this dense structure of the adsorption phase stabilized by sodium ions that is responsible for the high overpotential of the ascorbate oxidation. It not only retarded the ascorbate from occupying the reaction sites on the gold surface, but also making it difficult for water and $\mathrm{HPO}_{4}{ }^{2-}$, the potential proton acceptors, to accept protons released from the proton coupled electrochemical reaction.

\section{Results and discussion}

\section{The dynamic adsorption phase at the electrochemical interface}


We constructed the in situ three-electrode electrochemical cell with a gold film electrode serving as the working electrode and two platinum wires as the counter and reference electrodes, respectively, as shown in Figure 1a and Figure S1 in the Supporting Information. The gold film electrode was sputter-coated beneath a silicon nitride $\left(\mathrm{Si}_{3} \mathrm{~N}_{4}\right)$ membrane window on top of the liquid channel. More details for the assembly of the electrochemical microcell were given in the previous report. $^{22}$ The cyclic voltammetry $(\mathrm{CV})$ curve of the gold film electrode in the neutral phosphate buffer $(\mathrm{pH}=7.0)$ is shown in Figure 1b. A pair of peaks with the oxidation and reduction peak values at $+0.60 \mathrm{~V}$ and $+0.30 \mathrm{~V}$, respectively, are clearly visible, which were ascribed to the adsorption and desorption of the phosphate ions onto and from the gold electrode surface $^{17,29}$. To exclusively unravel the dynamic electrochemical interface chemistry that happened at the gold surface during the CV scanning in the phosphate buffer, we then interfaced the cells with the in situ liquid SIMS instrument which allowed the $\mathrm{Bi}_{3}{ }^{+}$ion beam to probe the $\mathrm{SiN} / \mathrm{Au}$ interface through a drilled small aperture of $\sim 2 \mu \mathrm{m}$ in diameter. As depicted in Figure 1a, the primary ion beam constantly bombarded the sidewall of the bowl-shaped aperture and thus ion fragments from the interface between the sidewall of the gold film electrode and the electrolyte diffused around the aperture was in situ generated and monitored by the mass spectrometer. The real-time chemical depth profiles in positive and negative ion modes during three consecutive CV scans were obtained and shown in Figures 2 and S2-S4. 

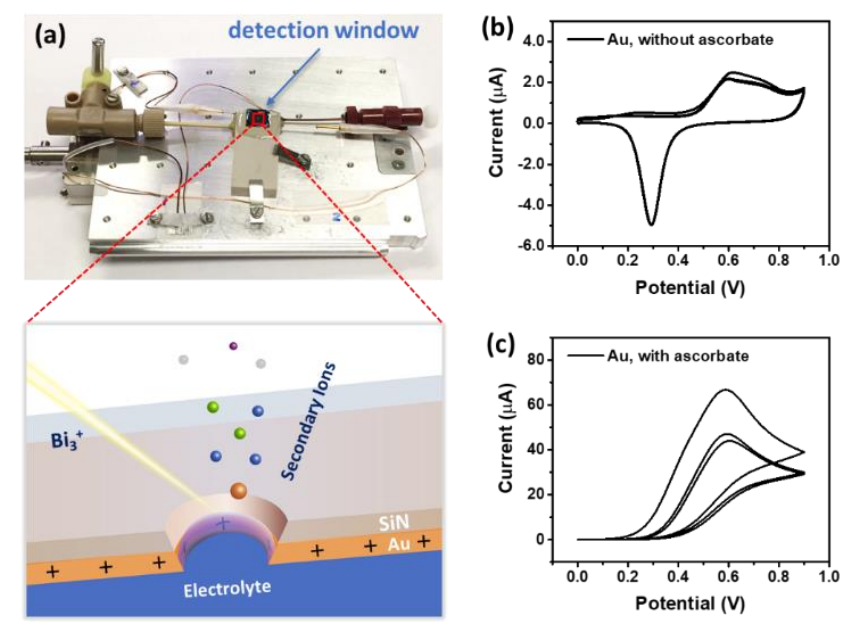

Figure 1. (a) A photo of the high vacuum-compatible electrochemical microcell assembled on a sample holder for in situ liquid SIMS analysis and an illustration of in situ monitoring of the electrode-electrolyte interface through the micro-aperture drilled by the primary $\mathrm{Bi}_{3}{ }^{+}$ions. $(\mathrm{b}, \mathrm{c})$ Cyclic voltammograms (CVs) obtained at the gold film electrode in $10 \mathrm{mM}$ phosphate buffer $(\mathrm{pH} 7.0)$ in the absence (b) and presence (c) of $10 \mathrm{mM}$ ascorbate in a fabricated microfluidic devices consisting of three electrodes with a gold film as WE and two Pt wires as RE and CE. Scan rate: $25 \mathrm{mV} \mathrm{s}^{-1}$.

The real time signals of representative ions arising from the phosphate buffer, $\mathrm{PO}_{3}{ }^{-}$at $\mathrm{m} / z$ and $\mathrm{Na}^{+}$at $m / z$ 23, were monitored at the electrode-electrolyte interface during the potential scanning (Figures $2 \mathrm{a}$ and $2 \mathrm{~b}$ ) under negative and positive ion modes, respectively. $\mathrm{PO}_{3}{ }^{-}$was the fragment ion of $\mathrm{H}_{2} \mathrm{PO}_{4}{ }^{-}$and $\mathrm{HPO}_{4}{ }^{-}$which are the dominant components of anions in the electrolyte at $\mathrm{pH}$ of 7.0 with the molar ratio of $\sim 1.6$ calculated according to the $\mathrm{p} K \mathrm{a}$ values of $\mathrm{H}_{3} \mathrm{PO}_{4}{ }^{11}$ The signal trend of $\mathrm{PO}_{3}{ }^{-}$as a function of the dynamic electrode potential showed an increase with elevating the potential in the anodic direction and a decrease with lowering the potential in the cathodic direction (Figure 2a). On the basis of the classic electrical double layer, it is easy to understand that the higher the electrode potential, the larger the number of negatively 
charged anions, such as $\mathrm{H}_{2} \mathrm{PO}_{4}{ }^{-}$and $\mathrm{HPO}_{4}{ }^{2-}$, were electrostatically attracted onto the gold surface as reflected by $\mathrm{PO}_{3}{ }^{-}$ion signal intensity. Besides, during dynamic $\mathrm{CV}$ scanning, we also detected $\mathrm{Au}\left(\mathrm{HPO}_{3}\right)^{-}$ions at $\mathrm{m} / \mathrm{z} 277$, of which the signal intensity fluctuated in a similar tendency to $\mathrm{PO}_{3}{ }^{-}$ ions (Figure S2a), further verifying the accumulation of $\mathrm{H}_{2} \mathrm{PO}_{4}{ }^{-}$and $\mathrm{HPO}_{4}{ }^{2-}$ anions on the anodic polarized electrode surface.

(a)

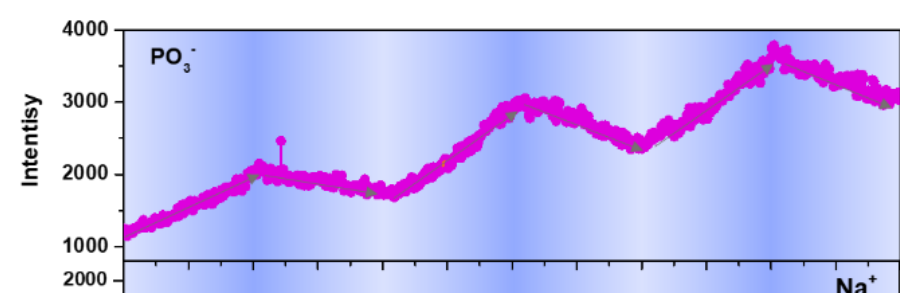

(c)

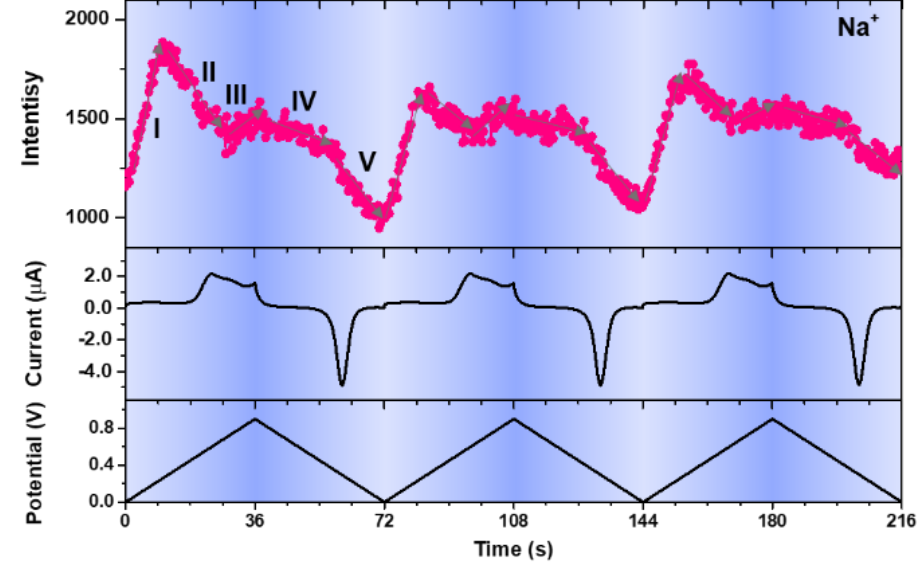

Figure 2. The potential-resolved signal trends of $\mathrm{PO}_{3}{ }^{-}$in the negative ion mode (a), $\mathrm{Na}^{+}$in the positive ion mode (b), and the dynamic electrochemical current (c) during the one representative potential scan (d) on the gold electrode surface in $10 \mathrm{mM}$ phosphate buffer.

What's extraordinarily interesting is that the trend of $\mathrm{Na}^{+}$ions did not show an opposite way to that of $\mathrm{PO}_{3}{ }^{-}$ions as expected, which instead presented a much more complex potentialdependence with five distinct regions during each dynamic potential scanning as marked with the gray arrows in Figure $2 b$. 
Surprisingly, in the first region $\mathbf{I}$ corresponding to potential range from 0 to $+0.28 \mathrm{~V}$, with elevating the electrode potential an abnormal increase of $\mathrm{Na}^{+}$ion abundance was observed. From an electrostatic point of view, attraction of cations onto positively charged electrode surface is impossible as the classic double layer theory predicts the repulsion of cations from the positively charged electrode surface by the Coulombic force, which results in a cation depleted inner Helmholtz layer. This theory has been experimentally verified by our previous liquid SIMS observation in $\mathrm{KNO}_{3}$ system where the $\mathrm{K}^{+}$ion signal detected at the gold-electrolyte interface dramatically decreased upon the anodic scanning ${ }^{24}$. Further evidence was reported in our recent liquid SIMS probing of a $\mathrm{Li}^{+}$-rich but anion-depleted inner Helmholtz layer at a negatively charged electrode surface in a Li ion battery. ${ }^{28}$ These results reflected that the probing depth of our operando liquid SIMS technique is only about one to two monolayers on the electrode surface. Therefore, the strong and rising $\mathrm{Na}^{+}$ion signal detected herein clearly showed that a large number of sodium ions existed within the inner Helmholtz layer, which could not be simply described by the classic double layer model. The detection of $\mathrm{AuNaH}_{2} \mathrm{PO}_{4}{ }^{-}$ions at $\mathrm{m} / z 317$ as well as $\mathrm{Na}\left(\mathrm{HPO}_{4}\right)_{2}{ }^{-}$ion clusters at $\mathrm{m} / z 215$ at the electrode-electrolyte interface (Figures S2b and S2c), of which the signal intensities elevated with the increasing potential in the anodic scan, revealed that sodium ions formed ion pairs with the concentrated phosphate ions on the gold electrode surface with positive surface charge. Moreover, unlike our previous observations of strong signals of hydrated ions, e.g. $\mathrm{K}_{(}\left(\mathrm{H}_{2} \mathrm{O}\right)_{\mathrm{n}}{ }^{+}$and $\mathrm{H}\left(\mathrm{H}_{2} \mathrm{O}\right)_{\mathrm{n}}{ }^{+}$, at the electrode-electrolyte interfaces in $\mathrm{KOH}$ or $\mathrm{KNO}_{3}$ system ${ }^{21,24}$, signal intensities of the hydrated sodium, $\mathrm{Na}\left(\mathrm{H}_{2} \mathrm{O}\right)_{n}{ }^{+}$ (Figure S3), and water cluster ions, $\left.\mathrm{H}_{(} \mathrm{H}_{2} \mathrm{O}\right)_{\mathrm{n}}{ }^{+}$(Figure S4), at the gold-phosphate electrolyte interface were very weak, and especially the signals of the water cluster ions did not show potential-dependent fluctuation behavior. These indicate that ions at the interface in this system 
appear to present in an at least partially dehydrated form, as a consequence, facilitating the formation of the compact cation-anion ion pairs at the interface. As illustrated in Figure 3-I, the existence of the cation-anion interactions seems to play a role in stabilizing the interfacial region where at positive electrode potentials the negatively charged ions were highly enriched and repulsion interactions might occur. With increasing the potential, more and more phosphate ions were attracted onto the gold surface. This required more sodium ions for specific cation-anion interactions to stabilize the interface that was crowded with the negatively charged ions, resulting in an increase in the $\mathrm{Na}^{+}$ion abundance in this region (I, Figure 2b).

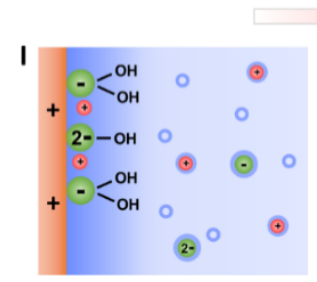

Electrical Attraction $\uparrow$

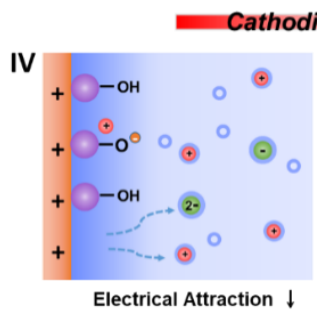

Anodic Direction

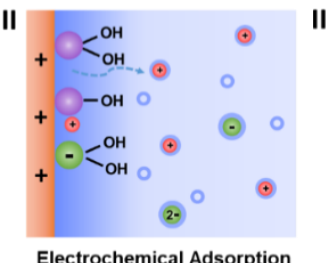

Electrochemical Adsorption
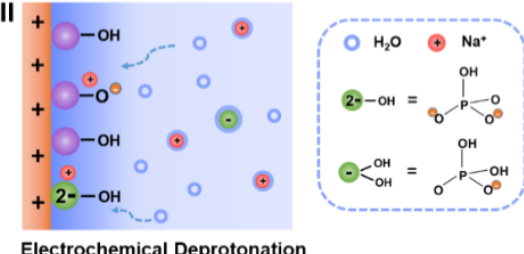
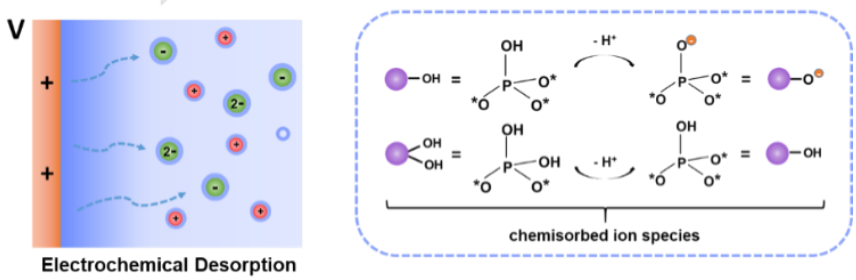

Figure 3. Diagrams of the dynamic adsorption phase at the electrochemical interface between a gold surface and a neutral phosphate buffer. $\mathrm{O}^{*}$ denotes the oxygen atom that is bond to the gold surface. (I) With elevating potential from 0 to $+0.28 \mathrm{~V}$, phosphate ions were electrically attracted onto the positively charged electrode surface, and surprisingly sodium ions coexisted in the inner Helmholtz layer forming compact ion pairs with phosphate ions. (II) With increasing the potential from +0.28 to $+0.60 \mathrm{~V}$, partial charge transfer occurred between the phosphate ions and the gold surface, which stabilized the adsorption phase and released some of sodium ions. (III) Within the region between +0.60 to $+0.90 \mathrm{~V}$, the high electrode potential resulted in the 
electrochemical deprotonation, the products of which showed higher affinity to $\mathrm{Na}^{+}$ions and slightly enhanced the $\mathrm{Na}^{+}$ion abundance. (IV) During the cathodic scan from +0.90 to $+0.45 \mathrm{~V}$, the electrical attraction force was decreased, leading to the partial release of the adsorption phase. (V) From +0.45 to $0 \mathrm{~V}$, the adsorption phase was electrochemically desorbed.

In region II $(+0.28 \sim+0.60 \mathrm{~V})$, the signal of $\mathrm{Na}^{+}$ions exhibited a sharp decrease in a manner opposite to that in the region I. In this region with further increase in the electrode potential an obvious increase in the anodic current was observed as shown in Figures $1 \mathrm{~b}$ and $2 \mathrm{c}$. The current increase was ascribed to the partial charge transfer during the electrochemical adsorption of phosphate ions that were attracted onto the gold surface to form the adlayer of the chemisorbed species, $\left(\mathrm{H}_{2} \mathrm{PO}_{4}\right)_{\text {ads }}$ and $\left(\mathrm{HPO}_{4}\right)_{\text {ads }}$. This chemisorption process stabilized the surface adlayer due to the direct bond formation between phosphate anions and the gold electrode surface. As a consequence, a portion of sodium ions were released from the compact ion pairs and repulsed by the higher electrode potential into the bulk electrolyte (Figure 3-II), leading to the decline in the $\mathrm{Na}^{+}$ion signal of the surface adlayer.

In region III $(+0.60 \sim+0.90 \mathrm{~V})$, with further increase in the potential the $\mathrm{Na}^{+}$ion abundance slightly rebounded. In a recent study of using the surface-enhanced IRAS, the chemisorbed phosphate ion species at the electrochemical interface was demonstrated to partially undergo a potential induced deprotonation at high electrode potentials. ${ }^{11}$ Our density functional theory (DFT) calculations also showed that after applying a higher electric field the $\mathrm{H}$ atoms appear to be pushed farther away from the gold surface as shown in the optimized geometries of both the adsorbed $\mathrm{H}_{2} \mathrm{PO}_{4}$ and $\mathrm{HPO}_{4}$ species (Figure S5). The deprotonation process shifted the acid-base equilibrium at the interface to lower $\mathrm{pH}$ compared to that of the bulk phosphate solution as depicted by the following equations (1-2). ${ }^{11}$ Although the higher the electrode potential, the 
stronger the repulsion for the $\mathrm{Na}^{+}$ions was caused by the Coulombic force, the $\mathrm{Na}^{+}$ions possess the higher affinity to the deprotonation products of the adsorbed phosphate species as exampled by the comparison between the optimized geometries of $\mathrm{NaH}_{2} \mathrm{PO}_{4}$ and $\mathrm{NaHPO}_{4}$ species on $\mathrm{Au}_{24}$ clusters (Figure S6). Taken together, these interactions contributed to the slight increase in the abundance of $\mathrm{Na}^{+}$ions at the electrode-electrolyte interface as shown in Figure $2 \mathrm{~b}$ and illustrated in Figure 3-III. However, earlier IRAS studies reported controversial results, ${ }^{30,31}$ some of which concluded that with the more positive polarization the adsorbed phosphate species underwent the protonation phenomenon. ${ }^{30}$ This discrepancy may be attributed to the interference of the spectral features by the bulk solution in the elucidation of the adsorbed phosphate species, which was overcome by the surface-enhanced IRAS technique. ${ }^{11}$ The chemical structure of the dynamic adsorption phase as revealed herein by our in situ liquid SIMS data as well as the DFT calculations supported the deprotonation phenomenon at high positive electrode potentials. The present work also implicates that the special attention should be paid to the experimental accuracy in elucidating the adsorbed species at the interface by employing the spectroscopy methods especially those which are not intrinsically surface-sensitive. With the virtue of high spatial resolution for the nanoscale presence of the double layer, the in situ liquid SIMS technique has the unique advantage of the high specificity and sensitivity of the chemical probing at the electrode-electrolyte interface, providing much more straightforward and convincing evidences into the nature and structure of the dynamic complex adsorption phase.

$$
\begin{aligned}
& \left(\mathrm{H}_{2} \mathrm{PO}_{4}\right)_{\mathrm{ads}} \rightleftharpoons\left(\mathrm{HPO}_{4}\right)_{\mathrm{ads}}+\mathrm{H}^{+} \\
& \left(\mathrm{HPO}_{4}\right)_{\mathrm{ads}} \rightleftharpoons\left(\mathrm{PO}_{4}\right)_{\mathrm{ads}}+\mathrm{H}^{+}
\end{aligned}
$$


In region IV which spans from +0.90 to $+0.45 \mathrm{~V}$ in the cathodic direction, with decrease in the potential the electrostatic attraction force to phosphate ions became weaker as evidenced by the signal trend of $\mathrm{PO}_{3}{ }^{-}$ions shown in Figure 2a. Some of the attracted phosphate species could be released from the gold surface into the bulk solution and therefore, part of the cation-anion interactions at the interface were broken (Figure 3-IV), leading to the gradual decrease in the signal intensity of $\mathrm{Na}^{+}$ions.

A steeper decrease in $\mathrm{Na}^{+}$ion signal was observed in region $\mathbf{V}$ ( +0.45 to $0 \mathrm{~V}$ ). On one hand, during the cathodic potential scanning over this relatively low potential range, the strength in the electrostatic attraction of the gold surface towards phosphate ions continued decreasing. On the other hand, a cathodic current peak appeared in this region (Figures $1 \mathrm{~b}$ and $2 \mathrm{c}$ ), which was attributed to the electrochemical desorption of the adlayer of phosphate species from the gold surface. During this desorption process, $\mathrm{Na}^{+}$ions in the compact ion pairs were released and repulsed away (Figure 3-V). The above two factors contributed to the steeper descent in the $\mathrm{Na}^{+}$ ion abundance in comparison with the trend in region IV.

To summarize the above results and discussion, the gold electrode surface in the phosphate buffer suffered from the strong adsorption of phosphate ions which was stabilized by the participation of sodium ions in the inner Helmholtz layer as evidenced by our in situ liquid SIMS data. The resulted electrochemical interface must cause influences on electrochemical reactions on gold surfaces when phosphate buffer serves as the electrolyte media.

\section{The role of the adsorption phase at the electrochemical interface}

To verify the role and influence of the absorption phase consisting of sodium-phosphate ion pairs described above on electrochemical reactions, we used in situ liquid SIMS to explore the 
electrochemical oxidation reaction of ascorbate at the gold electrode surface in phosphate buffer. Ascorbate is of considerable interest because of its multifunctional physiological functions in cerebral systems. It is not only neuroprotective as an antioxidant and free radical scavenger, but also serves as a neuromodulator of both dopamine and glutamate mediated neurotransmission in the central nervous system. ${ }^{10,32-34}$ The in vivo monitoring of its dynamic changes is very essential for probing the related brain chemistry and promoting the fundamental understanding in the molecular basis of brain functions. ${ }^{32,34}$ In the phosphate buffer, the electro-oxidation of ascorbate occurred with a peak current value at $0.59 \mathrm{~V}$, which is irreversible as no reduction peak was observed (Figure 1c). The high oxidation potential essentially causes difficulty in the selective electrochemical detection of ascorbate due to the interference from other electroactive neurochemicals coexisting in the in vivo system. ${ }^{32}$ Interestingly, the oxidation peak of ascorbate nearly overlapped with that of the ascorbate-free system (Figure 1b), indicating the link between the formation of the adsorption phase and the high overpotential of electro-oxidation.

We then performed in situ liquid SIMS measurements on the system containing ascorbate in real time and the obtained consecutive dynamic chemical depth profiles under positive and negative ion modes were shown in Figures S7-S11. Representative signals during one CV scan are shown in Figure 4. It can be seen that the electrochemically inert ion species in the electrolyte such as $\mathrm{H}_{2} \mathrm{PO}_{4}^{-} / \mathrm{HPO}_{4}{ }^{-}$which were reflected by the detection of $\mathrm{PO}_{3}{ }^{-}$ions (Figure $4 \mathrm{a}$ ), and $\mathrm{Na}^{+}$ ions (Figure 4b) exhibited characteristic potential-dependent behaviors. $\mathrm{The}^{+}{ }^{+}$ion signal decreased with increasing of potential in the anodic direction, and increased with decreasing of potential in the cathodic direction. The signal trend of $\mathrm{PO}_{3}{ }^{-}$ions was in a contrary way. These were in well accordance with the prediction of the classic electrical double layer theory, i.e. a highly positively charged electrode surface repulses positive ions and attracts negative ions. 
Comparing the potential-resolved signal changing of $\mathrm{Na}^{+}$ions in the ascorbate-containing system (Figure $4 b$ ) to that in the ascorbate-free system (Figure $2 b$ ), we noticed that the presence of ascorbate in the electrolyte distinctly changed the chemistry at the electrochemical interface. The pronounced decrease in the signals of $\mathrm{PO}_{3}{ }^{-}$and $\mathrm{Na}^{+}$ions, as well as the absence of $\mathrm{AuHPO}_{3}{ }^{-}$, $\mathrm{AuNaH}_{2} \mathrm{PO}_{4}{ }^{-}$and $\mathrm{Na}\left(\mathrm{HPO}_{4}\right)_{2}{ }^{-}$ions in the ascorbate-containing system (Figures $4 \mathrm{a}$ and $4 \mathrm{~b}, \mathrm{~S} 7-\mathrm{S} 8$ ) further revealed that a significant portion of the adsorption phase of phosphate ions stabilized by cation-anion interactions no longer existed. This conclusion was also strengthened by the appearance of the apparent signals of hydrated sodium ions and water cluster ions as shown in Figures S9-S10, the changes of which during the dynamic potential scanning were the same as that of sodium ions. Collectively, the above observations indicate that in order to be electrochemically oxidized on the gold surface the reactive ascorbate ions had to first break the adsorption phase and squeeze away the adsorbed non-reactive ion species that occupied the reaction sites on the gold surface.
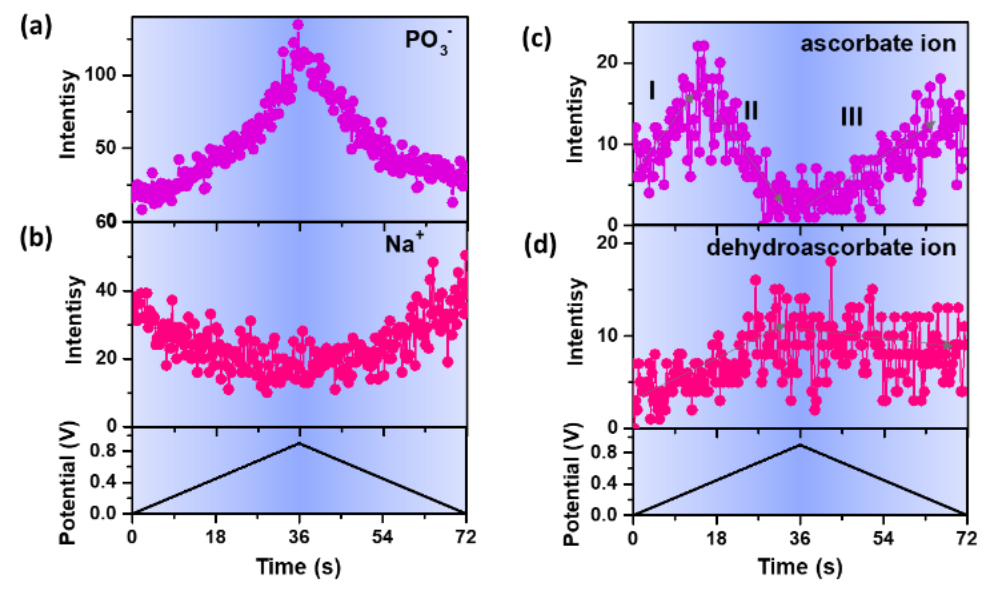

Figure 4. The potential-resolved signal trends of $\mathrm{PO}_{3}{ }^{-}$ion (a), $\mathrm{Na}^{+}$ion (b), the reactant ascorbate ion at $m / z 175$ (c), and the product dehydroascorbate ion at $m / z 173$ (d) observed during one 
representative $\mathrm{CV}$ scan on the gold electrode surface in $10 \mathrm{mM}$ phosphate buffer containing 10 $\mathrm{mM}$ ascorbate.

In addition, the ion intensities of the reactant, ascorbate ion at $\mathrm{m} / \mathrm{z} 175$, as well as the molecular ion of the dehydroascorbate ion product at $\mathrm{m} / \mathrm{z} 173$ in the ascorbate-containing system were also monitored as shown in Figures 4c, 4d and S11, respectively. For control experiments, the signals of the above two ions in the ascorbate-free system were shown in Figure S12. Three distinct regions were identified on the signal trend of the ascorbate ions (Figure 4c). In the I region, its signal intensity increased from $0 \mathrm{~V}$ to $+0.38 \mathrm{~V}$ during the anodic polarization, which was attributed to the electrostatic attraction of the negatively charged ascorbate ions onto to the electrode surface as illustrated in Figure 5-I. Meanwhile, very low signal of the product ions was detected (Figure 4d), corresponding with no oxidation current in this region. In range II from $+0.38 \mathrm{~V}$ to $+0.90 \mathrm{~V}$, the dynamic signal of ascorbate ions (Figure 4c) was in an opposite trend compared to that in the region I. The sharply declined signal was due to the electro-oxidation of ascorbate (Figure 5-II) which produced dehydroascorbate as verified by the increase in the product ion signal (Figure $4 \mathrm{~d}$ ). In the region III from $+0.90 \mathrm{~V}$ to $0 \mathrm{~V}$ in the cathodic direction, the signal of the ascorbate ions surprisingly reversed. This phenomenon was not straightforward to understand as the abundance of the negatively charged ascorbate ions should decrease with the decreasing in the electrode potential. The underlying reason was that during the anodic direction there was competition between ascorbate and phosphate ions for adsorption sites at the gold electrode surface. The occupation of ascorbate at the gold surface only squeezed away part of the adsorption phase as illustrated in Figure 5-I. The remaining part of the adsorption phase was not as dense as that in the ascorbate-free system and did not need the stabilization of sodium ions, thus the intensity of $\mathrm{Na}^{+}$ions within the adlayer simply decreased (Figure $4 \mathrm{~b}$ ). During the 
cathodic polarization (region III), the remaining part of the adsorption phase was desorbed and repelled from the gold surface as evidenced by the signal trend of $\mathrm{PO}_{3}{ }^{-}$shown in Figure 4a. This left the vacancies for the specific adsorption of ascorbate ions as illustrated in Figure 5-III, leading to the abnormal increase in the intensity of ascorbate ions in the region III (Figure 4c). These observations provide the first time the molecular evidence of the competition dynamics between electroactive and non-electroactive species at the electrochemical interface. More importantly, this explains why the oxidation potential range of ascorbate well correlated with that of the phosphate adsorption (Figures $1 \mathrm{~b}$ and 1c), suggesting that the adsorption phase on gold blocked the reaction sites for ascorbate and resulted in the high oxidation reaction barrier.

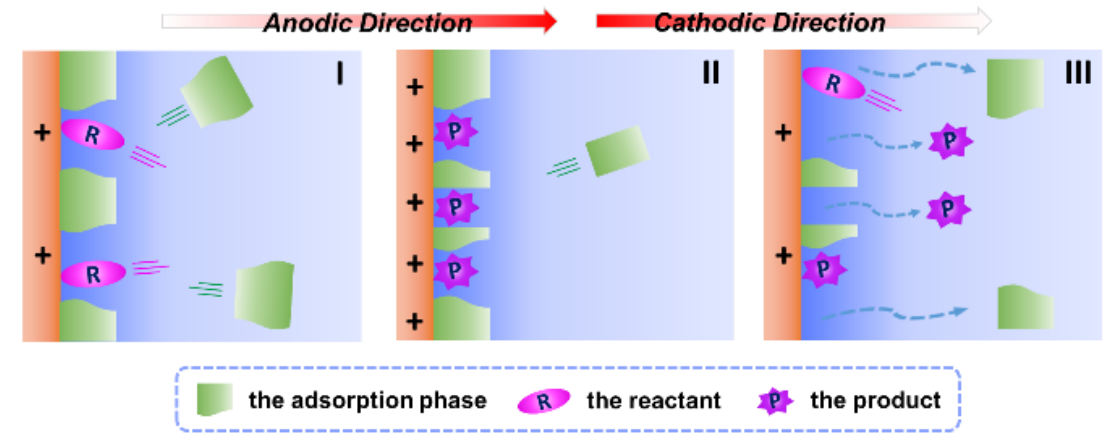

Figure 5. Diagrams of the dynamic electrochemical interface at a gold electrode surface in a neutral phosphate buffer containing ascorbate. (I) From 0 to $+0.38 \mathrm{~V}$ during the anodic direction, the added reactive ascorbate squeezed away a large part of the adsorption phase on the gold electrode surface. (II) In higher potential range from +0.38 to $+0.9 \mathrm{~V}$, the electrochemical oxidation reaction of ascorbate occurred, producing dehydroascorbate. (III) During the cathodic scan, the remaining part of the adsorption phase as well as the product were desorbed from the gold surface, leaving the vacancies for the specific adsorption of ascorbate ions. 
Furthermore, as evidenced from the product ion signal, the electro-oxidation of ascorbate is a typical proton coupled electron transfer reaction which involved the releasing of one proton and two electrons as show in Scheme S1, verifying the mechanism proposed in the previous report. ${ }^{10}$ It has been reported that individual water molecules or water clusters are proton donors or acceptors $^{35}$, which could serve as proton acceptors in the system herein. Besides, the phosphate species $\mathrm{HPO}_{4}{ }^{2-}$ from the supporting buffer could also act as a potential proton acceptor. ${ }^{36-38}$ The Hammes-Schiffer group revealed by DFT calculations that $\mathrm{HPO}_{4}{ }^{2-}$ is even favored over water as proton acceptor due to the higher base strength of $\mathrm{HPO}_{4}{ }^{2-}$ and the resulting shorter donoracceptor distance. ${ }^{37}$ According to our observations in the ascorbate-free phosphate buffer, less water molecules were available at the electrode-electrolyte interface due to the strong adsorption of phosphate species stabilized by forming ion pairs with $\mathrm{Na}^{+}$ions. Besides, the strong interactions between phosphate species and sodium ions in the ion pair may weaken the ability of $\mathrm{HPO}_{4}{ }^{2-}$ to act as proton acceptors. Therefore, during the proton coupled electrochemical oxidation of ascorbate, the ascorbate molecules broke the adsorption phase possibly not just fighting for the reaction sites on the gold surface. The underlying motivation may also include making the gold surface more wetting as well as setting the phosphate species free from the ion pairs in the adsorption phase and thus the water and $\mathrm{HPO}_{4}{ }^{2-}$ species could serve as proton acceptors at the interface. Therefore, the retarded proton transfer process caused by formation of phosphate-sodium ion pairs in the adsorption phase may be another reason of the high electrooxidation potential of ascorbate in this system.

The phosphate anion adsorption was known to cause the dramatic decrease in the electrochemical activity of metal surfaces in phosphate buffer, but the underlying molecular mechanism has long been obscure due to the challenge in unveiling the picture of the double 
layer structure. Our findings herein not only confirmed the adsorption of phosphate ions at the gold surface upon anodic polarization, but also revealed that the resulting adsorption phase was a dense layer though it was believed not to be easy because of the repulsive force imposed by the crowded negative-charged phosphate ions. Thanks to the high spatial resolution of liquid SIMS technique for the nanoscale characterization of the double layer, we unprecedentedly disclosed the existence of sodium cations in the inner Helmholtz layer in a partially dehydrated form. It is these sodium cations that hold hands with the highly concentrated phosphate ion species on the gold electrode surface, and knit a dense adsorption layer most possibly with a network-like structure as shown in Figure S13. In this case, the dense structure not only retarded the access of the electroactive species onto the gold surface, but also diminished the chances of water and phosphate species acting as proton acceptors at the interface. Therefore, it is anticipated that if the formation of the adsorption phase on gold surfaces in the phosphate buffer could be avoided or the structure be finely tuned, the ascorbate electro-oxidation might be promoted by speeding up electron transfer.

In classic double layer theory, positively charged ions would be repulsed away from an anodic polarized electrode surface. Therefore, the cations in the supporting electrolyte should have very limited effects on the anodic electrochemical reactions. However, the actual situation is much more complex. The traditionally considered "inert" cations from the supporting electrolytes have been reported to influence the rates and even the reaction selectivity of many electrochemical reactions in a complex manner of suppressing or promoting. ${ }^{39-44}$ For example, Marković and coworkers found that the catalytic activities of the hydrogen oxidation and methanol oxidation reactions on platinum in alkaline electrolytes increased when the cations going from $\mathrm{Li}^{+}$to $\mathrm{Cs}^{+}{ }^{39}$ They proposed a double layer structure that played a crucial role in this case, within which alkali 
cations might interact with the adsorbed $\mathrm{OH}$ species on the electrode surface. Being similar to our observations herein, apparently the "inert" cations in the supporting electrolytes are not as inert as we had expected. However, direct molecular evidence of the exclusive structure, dynamics and properties of the electrical double layer that could decipher the behaviors of cations has not been gained until our present work. In particular, knowledge of where and how the cations exactly reside in the double layer has been scarce. Therefore, computational studies, such as DFT calculations, were used to speculate the cation effects. ${ }^{39}$ To this end, with the merit of tracking in real time the exact position and dynamics of cations at electrode-electrolyte interfaces, our in situ liquid SIMS demonstrated herein provides a unique and indispensable solution to depict a more detailed and comprehensive picture of the double layer structure during operando conditions. This will reduce ambiguity into the interpretation of cation effects on electrochemical reactions.

\section{Conclusions}

In this work, we investigated the formation and evolution of the adsorption phase at an anodic gold electrode surface in the ubiquitous electrolyte system consisting of the multicomponent sodium phosphates by means of liquid SIMS. Our SIMS analysis at the electrochemical interface under operando conditions demonstrated that the phosphate anions were attracted onto the positively charged gold electrode surface as expected. Surprisingly, we probed the coexistence of sodium cations in the inner Helmholtz layer which formed ion pairs with the phosphate anions accumulated at the anodic polarized gold surface. We further unraveled that the traditionally considered "inert" cations from the supporting electrolyte participated in electrochemical reactions in subtle but impactful ways. It was the sodium cations that played a key role in formation of the strong and dense adsorption phase at the electrochemical interface. Apart from 
retarding the access of electrochemically active species onto the gold electrode surface, the dense layer made it difficult for water and $\mathrm{HPO}_{4}{ }^{2-}$ to act as proton acceptors during proton coupled electro-oxidation reactions. Both of the effects contributed to the high anodic potential to drive the oxidation of ascorbate in this system. This implicates that appropriate selection of cations in supporting electrolytes to circumvent or finely tune the unfavourable adsorption phase while remaining the access of active sites on the electrode surface can be a new effective and general strategy to promote electrochemical activities. We anticipate that this study brings fundamental insights for advancement of electrochemical systems in a widespread range of research and application fields including electro-catalysis, electrode sensing, and energy storage by rationally controlling the structure and property of the electrochemical interfaces.

\section{ASSOCIATED CONTENT}

Supporting Information. The following files are available free of charge. additional in situ liquid SIMS data and DFT calculation results (PDF)

\section{AUTHOR INFORMATION}

\section{Notes}

The authors declare no competing financial interests.

\section{ACKNOWLEDGMENT}

We thank the NSFC (Grant Nos. 21927804, 21904128, 21790390 and 21790392) and the National Key R\&D Program of China (Grant Nos. 2018YFA0800903) for support.

\section{REFERENCES}


1. Magnussen, O. M.; Groß, A., Toward an Atomic-Scale Understanding of Electrochemical Interface Structure and Dynamics. J. Am. Chem. Soc. 2019, 141 (12), 4777-4790.

2. Steinmann, S. N.; Wei, Z.-Y.; Sautet, P., Theory and experiments join forces to characterize the electrocatalytic interface. Proc. Natl. Acad. Sci. U.S.A. 2019, 116 (16), 7611.

3. Favaro, M.; Jeong, B.; Ross, P. N.; Yano, J.; Hussain, Z.; Liu, Z.; Crumlin, E. J., Unravelling the electrochemical double layer by direct probing of the solid/liquid interface. Nature Commun. 2016, 7 (1), 12695.

4. Zaera, F., Probing Liquid/Solid Interfaces at the Molecular Level. Chem. Rev. 2012, 112 (5), 2920-2986.

5. Devanathan, M. A. V.; Tilak, B. V. K. S. R. A., The Structure of the Electrical Double Layer at the Metal-Solution Interface. Chem. Rev. 1965, 65 (6), 635-684.

6. Grahame, D. C., The Electrical Double Layer and the Theory of Electrocapillarity. Chem. Rev. 1947, 41 (3), 441-501.

7. Yang, K.; Kas, R.; Smith, W. A., In Situ Infrared Spectroscopy Reveals Persistent Alkalinity near Electrode Surfaces during CO2 Electroreduction. J. Am. Chem. Soc. 2019, 141 (40), 15891-15900.

8. Du, H.-Y.; Chen, S.-C.; Su, X.-J.; Jiao, L.; Zhang, M.-T., Redox-Active Ligand Assisted Multielectron Catalysis: A Case of CoIII Complex as Water Oxidation Catalyst. J. Am. Chem. Soc. 2018, 140 (4), 1557-1565.

9. Gisbert, R.; García, G.; Koper, M. T. M., Adsorption of phosphate species on polyoriented $\mathrm{Pt}$ and $\mathrm{Pt}(111)$ electrodes over a wide range of pH. Electrochim. Acta 2010, 55 (27), $7961-7968$.

10. Zhang, M.; Liu, K.; Gong, K.; Su, L.; Chen, Y.; Mao, L., Continuous On-Line Monitoring of Extracellular Ascorbate Depletion in the Rat Striatum Induced by Global Ischemia with Carbon Nanotube-Modified Glassy Carbon Electrode Integrated into a Thin-Layer Radial Flow Cell. Anal. Chem. 2005, 77 (19), 6234-6242.

11. Yaguchi, M.; Uchida, T.; Motobayashi, K.; Osawa, M., Speciation of Adsorbed Phosphate at Gold Electrodes: A Combined Surface-Enhanced Infrared Absorption Spectroscopy and DFT Study. J. Phys. Chem. Lett. 2016, 7 (16), 3097-3102.

12. Wu, F.; Su, L.; Yu, P.; Mao, L., Role of Organic Solvents in Immobilizing Fungus Laccase on Single-Walled Carbon Nanotubes for Improved Current Response in Direct Bioelectrocatalysis. J. Am. Chem. Soc. 2017, 139 (4), 1565-1574.

13. Guo, S.; Yu, P.; Li, W.; Yi, Y.; Wu, F.; Mao, L., Electron Hopping by Interfacing Semiconducting Graphdiyne Nanosheets and Redox Molecules for Selective Electrocatalysis. $J$. Am. Chem. Soc. 2020, 142 (4), 2074-2082.

14. Habib, M. A.; Bockris, J. O. M., Adsorption at the Solid/Solution Interface: An FTIR Study of Phosphoric Acid on Platinum and Gold. J. Electrochem. Soc. 1985, 132 (1), 108-114.

15. Weber, M.; Nart, F. C.; de Moraes, I. R.; Iwasita, T., Adsorption of Phosphate Species on $\operatorname{Pt}(111)$ and $\operatorname{Pt}(100)$ As Studied by in Situ FTIR Spectroscopy. J. Phys. Chem. 1996, 100 (51), 19933-19938.

16. Kraack, J. P.; Hamm, P., Surface-Sensitive and Surface-Specific Ultrafast TwoDimensional Vibrational Spectroscopy. Chem. Rev. 2017, 117 (16), 10623-10664.

17. Niaura, G.; Gaigalas, A. K.; Vilker, V. L., Surface-Enhanced Raman Spectroscopy of Phosphate Anions: Adsorption on Silver, Gold, and Copper Electrodes. J. Phys. Chem. B 1997, 101 (45), 9250-9262. 
18. Li, J.-F.; Zhang, Y.-J.; Ding, S.-Y.; Panneerselvam, R.; Tian, Z.-Q., Core-Shell Nanoparticle-Enhanced Raman Spectroscopy. Chem. Rev. 2017, 117 (7), 5002-5069.

19. Zhu, Z.; Zhou, Y.; Yan, P.; Vemuri, R. S.; Xu, W.; Zhao, R.; Wang, X.; Thevuthasan, S.; Baer, D. R.; Wang, C.-M., In Situ Mass Spectrometric Determination of Molecular Structural Evolution at the Solid Electrolyte Interphase in Lithium-Ion Batteries. Nano Lett. 2015, 15 (9), 6170-6176.

20. Wang, Z.; Zhang, Y.; Liu, B.; Wu, K.; Thevuthasan, S.; Baer, D. R.; Zhu, Z.; Yu, X.Y.; Wang, F., In Situ Mass Spectrometric Monitoring of the Dynamic Electrochemical Process at the Electrode-Electrolyte Interface: a SIMS Approach. Anal. Chem. 2017, 89 (1), 960-965.

21. Zhang, Y.; Su, M.; Yu, X.; Zhou, Y.; Wang, J.; Cao, R.; Xu, W.; Wang, C.; Baer, D. R.; Borodin, O.; Xu, K.; Wang, Y.; Wang, X.-L.; Xu, Z.; Wang, F.; Zhu, Z., Investigation of Ion-Solvent Interactions in Nonaqueous Electrolytes Using in Situ Liquid SIMS. Anal. Chem. 2018, 90 (5), 3341-3348.

22. Zhang, Y.; Wang, J.-G.; Yu, X.; Baer, D. R.; Zhao, Y.; Mao, L.; Wang, F.; Zhu, Z., Potential-Dynamic Surface Chemistry Controls the Electrocatalytic Processes of Ethanol Oxidation on Gold Surfaces. ACS Energy Lett. 2019, 4 (1), 215-221.

23. Zhang, Y.; Zeng, W.; Huang, L.; Liu, W.; Jia, E.; Zhao, Y.; Wang, F.; Zhu, Z., In Situ Liquid Secondary Ion Mass Spectrometry: A Surprisingly Soft Ionization Process for Investigation of Halide Ion Hydration. Anal. Chem. 2019, 91 (11), 7039-7046.

24. Wang, J.-G.; Zhang, Y.; Yu, X.; Hua, X.; Wang, F.; Long, Y.-T.; Zhu, Z., Direct Molecular Evidence of Proton Transfer and Mass Dynamics at the Electrode-Electrolyte Interface. J. Phys. Chem. Lett. 2019, 10 (2), 251-258.

25. Hua, X.; Xia, H. L.; Long, Y. T., Revisiting a classical redox process on a gold electrode by operando ToF-SIMS: where does the gold go? Chem. Sci. 2019, 10 (24), 6215-6219.

26. Liu, Y. Y.; Zhang, S. Z.; Ying, Y. L.; Xia, H. L.; Hua, X.; Long, Y. T., Ion-Specific Effects on Hydrogen Bond Network at a Submicropore Confined Liquid-Vacuum Interface: An in Situ Liquid ToF-SIMS Study. J. Phys. Chem. Lett. 2019, 10 (17), 4935-4941.

27. Wang, J.-G.; Hua, X.; Xia, H.-L.; Long, Y.-T., Pore Confined Liquid-Vacuum Interface for Charge Transfer Study in an Electrochemical Process. Anal. Chem. 2019, 91 (5), 3195-3198.

28. Zhou, Y.; Su, M.; Yu, X.; Zhang, Y.; Wang, J. G.; Ren, X.; Cao, R.; Xu, W.; Baer, D. R.; Du, Y.; Borodin, O.; Wang, Y.; Wang, X. L.; Xu, K.; Xu, Z.; Wang, C.; Zhu, Z., Real-time mass spectrometric characterization of the solid-electrolyte interphase of a lithium-ion battery. Nat. Nanotechnol. 2020, 15 (3), 224-230.

29. Hoogvliet, J. C.; Dijksma, M.; Kamp, B.; van Bennekom, W. P., Electrochemical Pretreatment of Polycrystalline Gold Electrodes To Produce a Reproducible Surface Roughness for Self-Assembly: A Study in Phosphate Buffer pH 7.4. Anal. Chem. 2000, 72 (9), 2016-2021.

30. Ye, S.; Kita, H.; Aramata, A., Hydrogen and anion adsorption at platinum single crystal electrodes in phosphate solutions over a wide range of pH. J. Electroanal. Chem. 1992, 333 (1), 299-312.

31. Moraes, I. R.; Nart, F. C., Vibrational study of adsorbed phosphate ions on rhodium single crystal electrodes. J. Electroanal. Chem. 2004, 563 (1), 41-47.

32. Zhang, M.; Yu, P.; Mao, L., Rational Design of Surface/Interface Chemistry for Quantitative in Vivo Monitoring of Brain Chemistry. Acc. Chem. Res. 2012, 45 (4), 533-543.

33. Rice, M. E., Ascorbate regulation and its neuroprotective role in the brain. Trends Neurosci. 2000, 23 (5), 209-216. 
34. Cheng, H.; Li, L.; Zhang, M.; Jiang, Y.; Yu, P.; Ma, F.; Mao, L., Recent advances on in vivo analysis of ascorbic acid in brain functions. Trends Anal. Chem. 2018, 109, 247-259.

35. Weinberg, D. R.; Gagliardi, C. J.; Hull, J. F.; Murphy, C. F.; Kent, C. A.; Westlake, B. C.; Paul, A.; Ess, D. H.; McCafferty, D. G.; Meyer, T. J., Proton-Coupled Electron Transfer. Chem. Rev. 2012, 112 (7), 4016-4093.

36. Costentin, C.; Louault, C.; Robert, M.; Savéant, J.-M., The electrochemical approach to concerted proton-electron transfers in the oxidation of phenols in water. Proc. Natl. Acad. Sci. U.S.A. 2009, 106 (43), 18143.

37. Ishikita, H.; Soudackov, A. V.; Hammes-Schiffer, S., Buffer-Assisted Proton-Coupled Electron Transfer in a Model Rhenium-Tyrosine Complex. J. Am. Chem. Soc. 2007, 129 (36), 11146-11152.

38. Jackson, M. N.; Jung, O.; Lamotte, H. C.; Surendranath, Y., Donor-Dependent Promotion of Interfacial Proton-Coupled Electron Transfer in Aqueous Electrocatalysis. ACS Catal. 2019, 9 (4), 3737-3743.

39. Strmcnik, D.; Kodama, K.; van der Vliet, D.; Greeley, J.; Stamenkovic, V. R.; Marković, N. M., The role of non-covalent interactions in electrocatalytic fuel-cell reactions on platinum. Nat. Chem. 2009, 1, 466.

40. Stoffelsma, C.; Rodriguez, P.; Garcia, G.; Garcia-Araez, N.; Strmcnik, D.; Marković, N. M.; Koper, M. T. M., Promotion of the Oxidation of Carbon Monoxide at Stepped Platinum Single-Crystal Electrodes in Alkaline Media by Lithium and Beryllium Cations. J. Am. Chem. Soc. 2010, 132 (45), 16127-16133.

41. Sitta, E.; Batista, B. C.; Varela, H., The impact of the alkali cation on the mechanism of the electro-oxidation of ethylene glycol on Pt. Chem. Commun. 2011, 47 (13), 3775-3777.

42. Chen, X.; McCrum, I. T.; Schwarz, K. A.; Janik, M. J.; Koper, M. T. M., Co-adsorption of Cations as the Cause of the Apparent $\mathrm{pH}$ Dependence of Hydrogen Adsorption on a Stepped Platinum Single-Crystal Electrode. Angew. Chem. Int. Ed. 2017, 56 (47), 15025-15029.

43. Kuo, D.-Y.; Kawasaki, J. K.; Nelson, J. N.; Kloppenburg, J.; Hautier, G.; Shen, K. M.; Schlom, D. G.; Suntivich, J., Influence of Surface Adsorption on the Oxygen Evolution Reaction on IrO2(110). J. Am. Chem. Soc. 2017, 139 (9), 3473-3479.

44. Liu, E.; Li, J.; Jiao, L.; Doan, H. T. T.; Liu, Z.; Zhao, Z.; Huang, Y.; Abraham, K. M.; Mukerjee, S.; Jia, Q., Unifying the Hydrogen Evolution and Oxidation Reactions Kinetics in Base by Identifying the Catalytic Roles of Hydroxyl-Water-Cation Adducts. J. Am. Chem. Soc. 2019, 141 (7), 3232-3239. 